Article

\title{
Game-Theoretic Solutions for Data Offloading in Next Generation Networks
}

\author{
Muhammad Asif ${ }^{1}$, Shafi Ullah Khan ${ }^{1}$, Rashid Ahmad ${ }^{2}$ and Dhananjay Singh ${ }^{3, *}$ \\ 1 Institute of Information Technology, Kohat University of Science and Technology 26000 Kohat, Pakistan; \\ asif253026@yahoo.com (M.A.); skhan@kust.edu.pk (S.U.K.) \\ 2 Department of Physics, Kohat University of Science and Technology 26000 Kohat, Pakistan; \\ rashidahmad@kust.edu.pk \\ 3 Department of Electronics Engineering, Hankuk University of Foreign Studies, Yongin 449-791, Korea \\ * Correspondence: dsingh@hufs.ac.kr
}

Received: 5 June 2018; Accepted: 10 July 2018; Published: 25 July 2018

\begin{abstract}
In recent years, global mobile data traffic has seen an unprecedented increase. This is due to worldwide usage of smart devices, availability of fast internet connections, and the popularity of social media. The Mobile Network Operators (MNOs) are, therefore, facing problems in handling this huge traffic flow. Each type of traffic, including real-time video, audio, and text has its own Quality of Services (QoS) requirements which, if not met, may cause a sufficient loss of profit. Offloading of these traffics can be made more efficient so that values of QoS parameters are enhanced. In this work, we propose an incentive-based game-theoretic frame work for downloading data. The download of each type of data will get an incentive determined by the two-stage Stackelberg game. We model the communication among single Mobile Base Station $(M B S)$ and multiple Access Points $(A P s)$ in a crowded metropolitan environment. The leader offers an economic incentive based on the traffic type and followers respond to the incentive and offload traffic accordingly. The model optimizes strategies of both the $M B S$ and $A P S$ in order to make the best use of their utilities. For the analysis, we have used a combination of analytical and experimental methods. The numerical outcome characterized a direct process of the best possible offloading ratio and legalized the efficiency of the proposed game. Optimal incentives and optimal offloading was the achievement of our proposed game-theoretic approach. We have implemented the model in MATLAB, and the experimental results show a maximum payoff was achieved and the proposed scheme achieved Nash Equilibria.
\end{abstract}

Keywords: Mobile Network Operator (MNO); access point; Stackelberg approach; game theory; quality of service; Nash equilibrium; MATLAB

\section{Introduction}

Wireless cellular networks have replaced conventional networks due to their compatibility with fast wireless internet connections. These networks enhanced the worldwide use of smart devices and social media. Mobile communication technology also needs to evolve with time to cope with overload and the demands of end users such as high throughput and reduced delay. In fact, maintaining QoS is a challenging task [1].

The growth in mobile network connections, such as 2G, 3G, 4G Long Term Evolution LTE, and 5G, has recently seen an exponential increase. According to 'Corps Information Systems Control Officer (CISCO), forecast global mobile data traffic is expected to be 10.8 exabytes per month by 2018, which is an 18-fold increase since 2014 [2]. Multimedia traffic, such as images and video sharing, is even more popular due to the generalization of the $3 \mathrm{G}$ and $4 \mathrm{G}$ LTE networks. It is expected that $66.6 \%$ of the total traffic will be video related by 2017-2018 [3]. 
This increase in data traffic creates a challenge for the MNOs because of the shared and limited radio spectrum. One of the solutions could be to upgrade the radio access networks (RAN); however, this is quite expensive. Mobile customers, on the other hand, are experiencing long latencies and low throughput, especially during peak hours and in crowded metropolitan environments [4].

Although mobile data offloading has emerged as one of the alternative solutions, it lacks an intelligent design. A more suitable solution to the problem is to introduce an intelligent technique to mitigate the overload of cellular networks. Several offloading options are available, such as through WiFis, Femto-cells, WiMAX, opportunistic communication, and IP flow Mobilities. However, offloading through WiFi networks has gained more popularity due to their easy deployment, configuration, and usability by smart phones. In the year 2013 alone, mobile data traffic offloaded through WiFi accounted for $45 \%$ of data traffic, and it is expected to be $52 \%$ by 2018 [5].

Game theory is a branch of applied mathematics that provides a helpful tool in modeling the interface among autonomous choice makers. In this paper, we propose a game theoretic model for mobile data offloading based on the heterogeneous traffics in which the MBS offers an economic incentive depending on the type of traffic. Priority for the traffics, such as video, audio, and text, can be adjusted and based on a higher priority specific data type that is offloaded to a certain $A P$ in controlled amounts.

To alleviate the overload issue of a 3G LTE network, many technologies are used, but none is as effective as Mobile Data offloading MDO. In this work, we study the MDO through a third party WiFi and focus on the necessary economic incentives based adjustment between the MNO and Access Point Offloading (APOs) to achieve the optimal offloading. We used one MNO and many APs in the offloading scenario. The MNO offloads the different kinds of traffics, such as real-time traffic, video traffic, audio traffic, and text traffic. Each traffic has its own QoS requirements and the loss of QoS may cause the MNO a sufficient loss in profit. To enhance the values of QoS parameters for different kinds of traffic, offloading has to be made more systemic. In the existing literature, we found various economic-based frameworks existing between an MNO and APOs. However, there is no offloading algorithm that ideally controls the heterogeneous traffic with respective QoS requirements and achieves optimal offloading. In this work, we propose an incentive-based game-theoretic framework that determines the offload of the traffic types and enhances the QoS requirements. According to the model, the MNO offers incentives based on the traffic types, and then the AP follows the traffic priority approach and offloads the traffic from the MNO. Secondly, we analyzed the optimal deployment of the APs for offloading and the effect of an increase or decrease in the number of APs for offloading. The MNO offers various incentive rates for all types of traffic and $A P S$ use these rates to offload traffic. We analyze the offloading rate, which is a tradeoff for all types of traffic, and determined the optimal offloading. The mathematical model results represented a direct calculating process of the optimal traffic types based offloading ratio and validated the efficiency of the proposed game. Simulation results showed that the proposed model significantly improved the MNO's profit and $A P s^{\prime}$ offloading ratio for all types of traffic.

This manuscript is organized as follows: We have summarized the related literature in Section 2. In the next Section 3, the system model is outlined. The proposed game-based model for heterogeneous traffic using a two-stage Stackelberg approach is discussed in Section 4. In Section 5, simulation scenarios are presented, and finally, Section 6 concludes the paper and future work is given.

\section{Related Work}

Over the last few years, the cellular network got congested due to the introduction of smart phone devices and fast networks. This increase in traffic on the cellular network requires a strategy for offloading traffic through other optimal complementary networks. In Reference [6], authors have provided the overview of key technologies for the offloading of data. In Reference [7], data offloading techniques in cellular networks are summarized, and delayed and non-delayed offloading is discussed. WiFi offloading in heterogeneous networks is a hot topic and is studied by many researchers. 
For example, in Reference [8], the quantitative performance of 3G mobile data offloading through WiFi and the test of performance through several smart phones that use WiFi network for offloading is presented. The results show a good offloading ratio.

In Reference [9], the authors considered the WiFi technology for offloading to reduce the load on the 4G-LTE network. The architectural aspect of mobile data offloading from the LTE to trusted WiF $i$ access networks is also discussed, and the performance parameters for offloading are included in the study. In Reference [10], the overload issue of the cellular networks, which arise due to the introduction of high-end devices and fast networks, is investigated. The authors considered integrated architecture exploiting the opportunistic networking paradigm to migrate data traffic from cellular networks to metropolitan WiFi access points (APs). In Reference [11], the offloading issue through WiFi technology for cellular networks and the usage based charging model is given. The proposed scheme shows the low burden on the base station (BS). In Reference [12], the authors discussed the issue of the spectrum utilization of the WiFi and proposed a resource allocation scheme that is based on a joint-user transfer using Nash Bargaining (NB) theory.

Mobile data offloading through WiFi requires a good understanding of economic benefits. To obtain the satisfactory rewards from traffic offloading is a complex issue for mobile customers. The economic aspect of mobile data offloading between microcell and small cells requires a cooperation and negotiation scheme. However, to obtain optimal offloading, the study of computation is important for researchers. In literature, we study many former approaches based on the economics issue between macro cells and small cells. In Reference [13], the authors consider the offloading through WiFi based on a two-stage sequential game. The results show that it is beneficial for both providers and users. In Reference [14], the authors designed a multileader and follower game based on the Stackelberg approach that sets the optimal price scheme for users. In Reference [15], a distributed market framework for offloading services is designed, which formulates a multi-leader multi-follower Stackelberg game (MLMF-SG). The experimental results show the existence of the equilibrium. In Reference [16], an incentive framework that investigates the tradeoffs between offloaded traffic and user satisfaction is proposed. The authors consider the parameters of incentive cost and delay tolerance. The experimental results show a greater efficiency in offloading.

The authors in Reference [17] address the MNO profit maximization problem and designed the incentive framework based on a three-stage Stackelberg game model. The experimental results show that equilibrium market price decreases. In Reference [18], the authors take the issue of resource allocation and power allocation in small cells and designed an algorithm based on the cooperative NB game theory. The authors claim to have results for the efficient and fair allocation in small cellular networks. In Reference [19], the authors take the issue of the front hauling in 5G Long term Ultra dense cloud small cell network (LTE-U UDCS) Nets and surveys the various technologies with its advantages and disadvantages for the front haul. The mechanism proposed in Reference [20] solves the energy efficiency issue. In this work, the authors designed a reverse auction-based Green Offloading (GO) scheme. The proposed scheme results achieve a high QoS.

The game theoretic solution for the overloading problem in Internet of thing (IoT) that are based on the Vickrey-Clarke-Groves (VCG) mechanism and Rubinstein bargaining game model is developed in Reference [21]. Mobile data offloading through WiFi is discussed in Reference [22], and the authors point out the practical issues faced by the MNOs, and also discussed the possible solution of these issues. Some researchers discussed the economic issue between the APs and the MNO [23]. To enhance the QoS, the authors designed an algorithm that is based on the double-auction mechanism that maximizes the network operation. The experiment results show that the proposed scheme achieves a good offloading decision and good revenue results. In Reference [24], authors have discussed the interaction between the MNO and APs with the help of Nash bargaining theory. The bargaining game produces the best economic solution for both the MNO and the APs. The multi-channel assignments are done in Reference [25], giving a high throughput and lower energy consumption. In Reference [26], the authors take the issue of the efficient power allocation in the uplink of two-tier closed-access 
Femtocell networks. The raised issue is addressed through a game-based algorithm that uses the network utility maximization theory. According to this theory, each mobile user is linked with an appropriately defined QoS-aware utility function and further gives higher priority to the Femtocell users and macrocell users. The users are also punished through a convex cost function with respect to their uplink transmission power.

To address the severe issues in heterogeneous cellular networks, such as Internet content caching, resource allocation, and computation offloading, an intelligent decentralized algorithm called alternating direction method of multipliers ADMM for computation offloading has been designed [27]. In experiments, the authors take various system parameters, and the results show a good performance of the proposed mechanism. In similar work, an ADMM-based distributed algorithm [28] addresses four basic issues, i.e., content caching, MEC computation resource allocation, spectrum resource allocation, and computation offloading decisions in the cellular networks. In Reference [29], the authors take the issue of the resource allocation algorithm and survey the various resource allocation algorithms based on various classes and highlight the pros and cons of each algorithm. They also discuss the various future research directions.

\section{System Model}

We considered a crowded metropolitan area where presumably most of the mobile users had smart phones and tablets for social networking. To achieve better service, a heterogonous network was formed, which consisted of the MBS and WiFi comprising a two-layer network. We had also assumed that the MBS covered a larger area as compared to the area covered by WiFi, Access Point $(A P)$, and both networks transmitted on different paths.

In a simplified scenario, as shown in Figure 1, we considered only one MBS and multiple APS in order to model a dense deployment scenario of heavy traffics. The total area covered by one $M B S$, where $N$ was the number of WiFi routers that were lying is given by:

$$
A_{M B S}=S_{M B S}-\sum_{K=1}^{N} S_{w i}
$$

where the areas of MBS and WiFi were $S_{M B S}$ and $S_{w i}$, respectively, and $i=1,2$. NAPS and the WiFi settings were organized in a way that there are no overlapped areas between them. In this crowded area, various mobile users used the network setup for many applications, and traffics generated by the mobile users could be offloaded to an $A P$ if the Mobile User (MU) was located within the converging area of the $A P$ and had the WiFi interface. The traffic profile of the MBS was given by:

$$
\begin{gathered}
L=\left\{L_{0}, L_{1}, \ldots, L_{N}\right\} \\
C_{m} T l_{m} \leq C_{m} T
\end{gathered}
$$

In Equation (2) the L represent the traffic load of access point. We defined a game-theoretic model for an offloading mechanism of APs. In this model, every AP tried to maximize the offloading ability. We, however, focused on the specific times, such as peak-time offloading and off-peak-time offloading, and our offloading game could be adopted at different traffic conditions. For the fixed time period each WiFi has an instantaneous rate $C_{m}$, where $m$ shows the size of $W i F i$, and hence the maximum amount of data that WiFi can serve within a certain time period is $C_{m} T$.

The throughput of WiFi network and throughput of the MBS network could be calculated as:

$$
\text { Throughput }_{M B S}=E\left(\text { Thro }_{M B S}\right) N_{M B S}
$$


and the throughput of the WiFi networks was given by:

$$
\text { Throughput }_{w i f i}=\sum_{K=1}^{N A P} E\left(\text { Throughput }_{w i f i}\right) N_{w i f i}
$$

The total throughput of the heterogonous network was the summation of both networks:

$$
\text { Throughput }=\text { Throughput }_{M B S}+\text { Throughput }_{w i f i}
$$

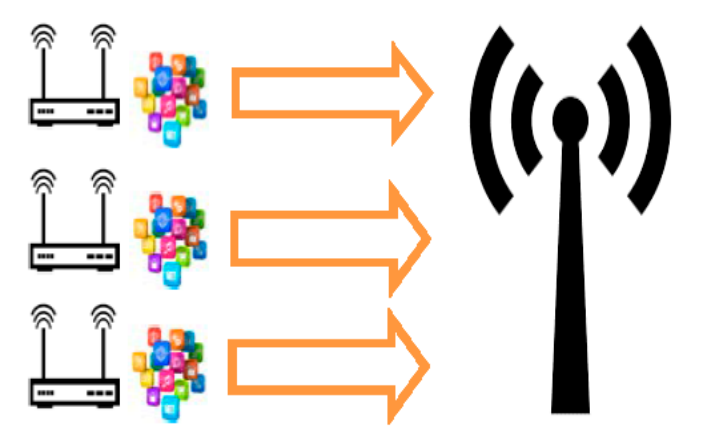

Figure 1. System Model.

\section{Game-Theoretic Model for Heterogeneous Traffic Using a Two-Stage Stackelberg Approach}

We proposed a Stackelberg follower and leader game in which the aim of the player is to maximize the APs overall payoff. The set of players consisted of three different kinds of APs depending on which type of traffic they download. Our game-theoretic model generated the heterogeneous traffic communication, such as video, audio, and text traffic, and these traffics are generated based on the particular traffic downloading priority:

$$
P=\left\{A P_{m V}, A P_{m A}, A P_{m T}\right\}
$$

where $V$ represents video, $A$ represents audio, and $T$ represents text traffic, and $m$ shows the number of $A P s$. The payoff function was given by:

$$
U_{m(1)}=\sum_{D} \beta_{D} \frac{l_{D}}{\sum_{m \in P} l_{n}}-\sigma_{m D} l_{m D}
$$

where $D=\{V, A, T\}$, and $V$ denoted the incentive offered for video traffic, $A$ was an incentive offered for audio traffic, and $T$ was the incentive offered for the text traffic. Further, $m V$ was the mobile user pay cost for video traffic, $m A$ was the mobile user pay cost for audio traffic, $m T$ was the mobile user pay cost of the text traffic, and $l_{v}, l_{A}$, and $l_{T}$ are the offloaded traffics.

To analyze the performance of the model, the payoff function was defined based on incentives offered by the MBS and the ability of $A P S$ to admit traffic downloads. The game was a noncooperative game where each $A P$ competes with each other to maximize its payoff function. The game had the following elements:

$$
G=\left(P,\left(S_{m}\right)_{m \in P},\left(U_{m \in P}\right)\right)
$$

where $G$ represented the game for offloading and $P$ represented players involved in the game, namely $M B S$ and $A P s,\left(S_{m}\right)_{m \in P}$ represented the strategy that we apply to get the maximum payoff and $\left(U_{m \in P}\right)_{m \in p}$ represented the payoff function set. 
Definition 1. The APs bid vector has three components one for each type of traffics such as video, audio, and text:

$$
l=\left(l_{1} \ldots l_{Q}, l_{1} \ldots l_{R}, l_{1} \ldots l_{S}\right)
$$

This bid vector is a Nash equilibrium of the offloaded game $G=\left(P,\left(S_{m}\right)_{m \in P},\left(U_{m \in P}\right)\right)$.

For all $m \in P, U_{m}\left(l_{m}, l_{-m}\right) \geq U_{m}\left(l_{m}, l_{-m}\right)$ for $l_{m} \geq 0$, where $\left(l_{m}, l_{-m}\right)$ is the resulting bid for the $m$ player given the other player's bid results $l_{-m}$.

Theorem 1. The game proposed here is for offloading the data prioritized based on the type of data. For example, in a particular scenario, it is possible to make video data downloaded on priority as compared to text data, and so on. We suppose that in such an offloading game, Nash equilibrium exists. The proof of this theorem goes as in Reference [30]. However, the bid vector is composed of three linearly independent components $l_{v}, l_{A}$, and $l_{T}$, and the strategy set $S$ is more complicated. It contains strategies which might depend upon one or more components of the bid vector.

Proposition. In the proposed game given by $G=\left(P,\left(S_{m}\right)_{m \in P},\left(U_{m \in P}\right)\right)$, a Nash equilibrium exists for all if:

(1) $\left(S_{m}\right)_{m \in P}$ is a nonempty, convex, and compact subset of a 3-dimensional Euclidean space,

(2) $U_{m}$ is continuous in $l$ and concave in $l_{m}$.

The defined strategy $S=\left\{\left|S_{m}\right|_{m \in P}: l_{m}\left|0 \leq l_{m} \leq C_{m}\right|\right\}$.

Space is a nonempty, convex, and compact subset of the Euclidean space.

To prove its concavity, we see that $U_{m}$ is obviously continuous in $l$ we take the second order derivative with respect to $l_{m}$.

The first derivative is given in the following:

$$
\frac{\partial U_{m}}{\partial l_{m}}=\sum_{D=V, A, T} \beta_{D}\left[\frac{\sum_{n \in p, n \neq m} l_{n}}{\sum_{m \in p} l_{n}^{2}}\right]-\sigma_{m D}
$$

For the second derivative with respective to $l_{m}$ we have:

$$
\frac{\partial^{2} U_{m}}{\partial^{2} l_{m}}=\sum_{D=V, A, T} \frac{-2 \beta \sum_{n \in p, n \neq m}^{n} l_{n}}{\sum_{n \in p}\left(l_{m}^{3}\right)}<0
$$

From the second order derivative, we see that $U_{m}$ is concave in $l_{m}$. The Nash equilibrium exists in the offloading game.

Theorem 2. In our case, the multiple noncooperative offloading games have a unique Nash equilibrium, since our game has in principle three components. We have shown in the theorem-1 that all the components have a Nash equilibrium. Let the three-component vector 1 denote the Nash equilibrium in the game, which has to satisfy $l=\operatorname{br}(l)$.

where: $b r(l)=b r_{l}(l), \ldots, b r_{q}(l), b r_{l}(l), \ldots, b r R(l), b r_{l}(l), \ldots b r S(l), b r_{m}(l)$ is the best response function of all the players, and $b r_{m}(l)=b r_{m}\left(l_{-m}\right)$.

Therefore, the $b r(l)$ is a standard function since it is positive, monotonic, and scalable [30].

Therefore, the defined game has a unique Nash equilibrium because the fixed point $l=b r(l)$ is unique for a standard function.

We now also argue that our best response of the game exists in this general setting. This optimal solution is the best response for the game as the total downloaded traffic for any AP, which is the sum of all types of data, i.e., text, audio, and video cannot exceed the total capacity of the AP. However, a further constraint can be imposed where we do not allow a particular type of traffic to exceed its capacity.

$$
\sigma_{m D} \sum_{D=V, A, T} l_{m D}<\beta_{D}
$$


This constraint can give us further control of the downloading options analyzed in detail in the simulation section below.

Therefore, the best response correspondence $b r_{m}(l)$ satisfies the three conditions of scalability, mono-tonicity, and positivity. Therefore, in our offloading game, there exists a unique Nash equilibrium

$$
G=\left(P,\left(S_{m}\right)_{m \in P},\left(U_{m}\right)_{m \in P}\right)
$$

Theorem 3. The total unique equilibrium for the game comprised of all types of data is given by:

$$
\sum_{D=V, A, T} l_{m D}^{*}=\left[\frac{\sum_{D=V, A, T} \beta_{D}(M-1)}{\sum_{n \in P} l_{n}} \times\left[1-\frac{(M-1) \sum_{D=V, A, T} \sigma_{m D}}{\sum_{n \in P} \sum_{D=V, A, T} \sigma_{m D}}\right]\right]_{0}^{C_{m D}}
$$

The offloading game is a two-stage game where the leader offers the incentive and followers offload the traffics based on the incentive.

Mobile Base Station (MBS) Strategy

The overall payoff function of the MBS was as follows:

$$
U_{M B S}\left(\sum_{D=V, A, T}^{n} \beta_{D}\right)=\delta \sum_{m \in p} l_{m D}-\sum_{D=V, A, T} \beta_{D} \sum_{m \in p} l_{m D}
$$

In Equation (16), the overall utility of the APS that was achieved in the offloading from the MBS and $\sum_{D=V, A, T} \beta_{D}$. $D$ was the economic incentives for the offloading traffic.

In our offloading game, which was a noncooperative-type game based on the APs utility, the objective of the MBS was to maximize its net profit by solving the optimization problem:

$$
\underset{\beta \leq \beta^{\max }}{\operatorname{maximize}_{\beta} U_{M B S}(\beta)}
$$

We obtained a maximized net profit:

$$
\max \left[\frac{\left(\delta-\sum_{D=V, A, T} \beta_{D}\right) \beta \sum_{D=V, A, T} \beta_{D}(M-1)}{\sum_{m D \in P}^{n} \sigma_{m D}} \times \sum_{m D \in P}\left(1-\frac{(M-1) \sigma_{m D}}{\sum_{m D \in P}^{n} \sigma_{m D}}\right)\right]
$$

from the Equation (16); we know that it is the convex optation problem, and its solution requires some standard convex optimization algorithms.

Here we considered the substitute option of the payoff function of MBS as follows:

$$
\begin{aligned}
U_{M B S}\left(\sum_{D=V, A, T} \beta_{D}\right) & =\delta \sum_{m \in p}^{n} \log \left(l_{m D}\right)-\sum_{D=V, A, T} \beta_{D} \sum_{m \in p} l_{m D} \\
& =\delta \log \left(\prod_{m D \in P} l_{m D}-\sum \beta_{D} \sum_{m \in p}^{n} l_{m D}\right.
\end{aligned}
$$

To achieve the proportional fairness offload among $A P S$, we shall use the logarithm utility function. We obtained a function when we substituted Equation (16) into the MBS utility function (17).

$$
\max \left[\delta \log \left[\left(\sum_{D=V, A, T} \beta_{D}\right)^{M} \Gamma\right]-\left(\sum_{D=V, A, T} \beta_{D}\right)^{2} \varphi\right]
$$


where

$$
\Gamma=\prod_{m D \in P} \frac{(M-1)}{\sum_{m D \in P}^{n} \sigma_{m D}}\left[1-\frac{(M-1) \sigma_{m D}}{\sum_{m D \in P}^{n} \sigma_{m D}}\right]
$$

and

$$
\varphi=\frac{(M-1)}{\sum_{m D \in P}^{n} \sigma_{m}}\left[1-\frac{(M-1) \sigma_{m D}}{\sum_{m D \in P}^{n} \sigma_{m D}}\right]
$$

We saw that the MBS utility function was concave, so by the first order optimality condition, we can obtain the optimal value of economic incentive $\beta^{*}$ :

$$
\left(\sum_{D=V, A, T} \beta_{D}\right)^{*}=\left[\sqrt{\frac{\delta M}{2 \varphi}}\right]^{\left(\sum_{D=V, A, T} \beta_{D}\right)^{\max }}
$$

The Lagrangian of the problem then became:

$$
L\left(\sum_{D=V, A, T} \beta_{D} \lambda\right)=\delta \log \left[\left(\sum_{D=V, A, T} \beta_{D}\right)^{M} \Gamma-\left(\left(\sum_{D=V, A, T} \beta_{D}\right)^{2} \varphi+\lambda\left(\sum_{D=V, A, T} \beta_{D}\right)-\left(\sum_{D=V, A, T} \beta_{D}\right)^{\max }\right]\right.
$$

In the above equation, $\lambda$ is the Lagrange multiplier.

Also, we knew that $\sum_{D=V, A, T} \beta_{D}=\beta_{V}+\beta_{A}+\beta_{T}$. Then

$$
\begin{aligned}
& \text { subjectto } \lambda \geq 0 \\
& \text { maximize } D(\lambda)
\end{aligned}
$$

The dual function was:

$$
D(\lambda)=\max _{\sum D=V, A, T} \beta_{D} L\left(\sum_{D=V, A, T} \beta_{D}, \lambda\right)
$$

With the help of the sub-gradient method, the dual function could be solved, which updated the Lagrange multiplier as follows:

$$
\lambda(t+1)=\left[\lambda(t)+\kappa(t)\left(\sum_{D=V, A, T}^{n} \beta_{D}(t)\right)-\left(\sum_{D=V, A, T}^{n} \beta_{D}(t)\right)^{\max }\right]^{+}
$$

where $k(t)$ is the step size at iteration $t$, which must be positive, and $[X]+=\max 0 ; X$. The optimal economic incentive $\beta^{*}$ could be obtained using the Karush-Kuhn-Tucker (KKT) condition.

$$
\frac{\delta L(\beta, \lambda)}{\delta \beta}=0
$$

The optimal solution of the economic incentive was as follows:

$$
\left(\sum_{D=V, A, T} \beta_{D}\right)^{*}=\left[\frac{\lambda+\sqrt{\lambda^{2}+8 \delta M \varphi}}{4 \varphi}\right]
$$

\section{Experiments, Results, and Discussion}

The proposed model did not exist in any practical form. Since the work completely relied on the additional functionality of $A P S$ and $M B S$. Therefore, these must be programmed accordingly to realize the practical implementation of this mechanism.

The experiments were conducted by considering a dense HetNet having a typical 3G/4G LTE macro cell. The transmission range of this macro cell was $500 \mathrm{~m}$. To offload traffics from $3 \mathrm{G} / 4 \mathrm{G}$ LTE, four APs are deployed. The transmission range of each $A P$ hotspot was $50 \mathrm{~m}$. The maximum mobile users (MUs) use their smartphone for downloading and uploading various traffics types. 
The proposed scheme, referred to as Game Theoretic Mobile Data Offloading (GMDO) in this section, has been compared with Economic Incentive for Data Offloading (EIDO) [30]. Data traffic for four major types of the applications, i.e., file backup, HTTP, video, and VoIP were considered in the experiments. For offloading, we considered both peak time and off-peak time testing. To conduct these experiments, we used MATLAB under MS Windows. The key parameters used for simulation are listed in Table 1.

Table 1. Values of Parameters.

\begin{tabular}{cc}
\hline Parameters & Value \\
\hline$M B S$ & 01 \\
MBS Bandwidth & $20 \mathrm{MHz}$ \\
Area of the $M B S$ & $1000 \times 1000 \mathrm{~m}^{2}$ \\
The total transmission power of $M B S$ & $46 \mathrm{dBm}$ \\
$A P s$ number in hotspot & 04 \\
Area of the $A P S$ & $100 \times 100 \mathrm{~m}^{2}$ \\
The total transmission power of $A P S$ & $30 \mathrm{dBm}$ \\
Number of users per & 80 \\
\hline
\end{tabular}

In Figure 2, the economic incentives were kept for all types of data equal to 1, i.e., $\beta_{D}=1$. We observed that the text data had the highest rate of the download. Audio and video data had comparatively lower rates, while the real data is the lowest among all types of data. However, Figure 2 shows that the downloaded traffic had an exponential increase when the numbers of piccolos were less than five and increasing. However, it stabilized for a higher and higher number of cells.

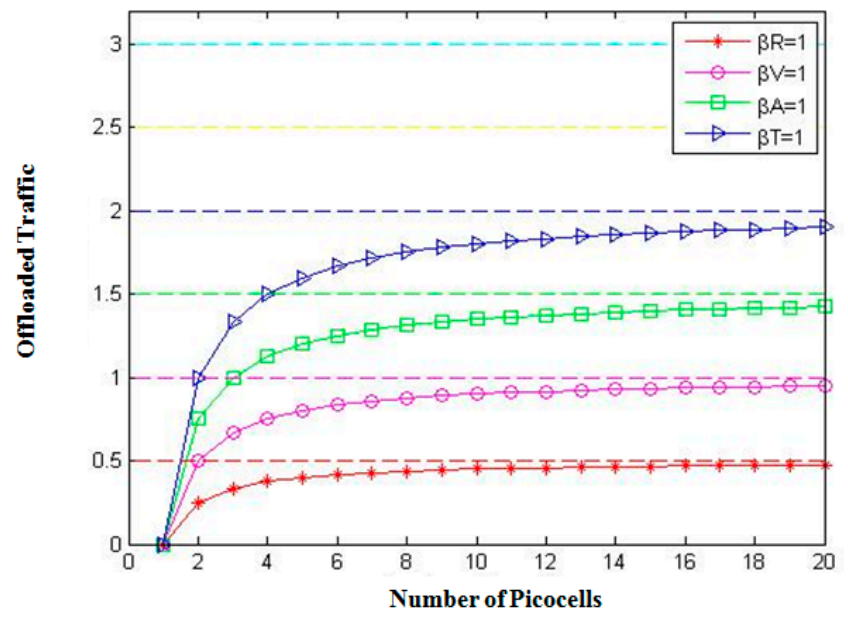

Figure 2. Offloaded traffic with) $\beta_{D}=4$.

The results of Figure 2 are then summarized in Table 2. As discussed earlier, the incentive ratio was given to different types of data, and the resultant graph Figure 2 shows the retrospective changes when we used 1 as the standard value of incentive for all four data types. The graph values rendered adjacent to some specific level, which is due to predefined extents for offloading of the particular data type.

Here, the real-time data graph line varied from 0 to 0.5 because least offloading occurred at a particular rate of incentive as compared to others. Video data showed a quicker response to the same ratio of incentives as compared to real-time data and still much less than the other two, i.e., audio and textual data types. Audio data had less importance as compared to video watching, therefore, the comparison result of the particular rate of incentive was much higher. The textual data offloading ratio is highest among all because of two reasons; first is its least size, while the second was not noticing 
the minor interruption. Primary channel allocation to the resources of greater importance was key to the comparison made in this model, and the secondary channel was considered reserved for offloading in cases to resolve the problem of congestion and to ensure increased throughput. A synopsis of the above discussion is as follows:

Table 2. Summary of Results of Figure 2.

\begin{tabular}{cccc}
\hline Data Type & Incentive Effect & Need for Offloading & Graph Variation \\
\hline Real-time data & Lowest & Least & Lowest \\
Video data & Low & Comparatively not necessary & Low \\
Audio data & Medium & Necessarily required & Medium \\
Textual data & High & Needed & High \\
\hline
\end{tabular}

A slight change was observed when real-time data was awarded an offloading ratio of 1.5 and its graph points toward some higher level, but still at the lowest level, while the remaining types are kept constant as shown in the Figure 3. The offloading ratio of real-time data got a position almost equal to the graph line of the video data when the rate was doubled. This strategy illuminated the minimum offloading ratio in real-time data, which had been illustrated in both parts (a) and (b).

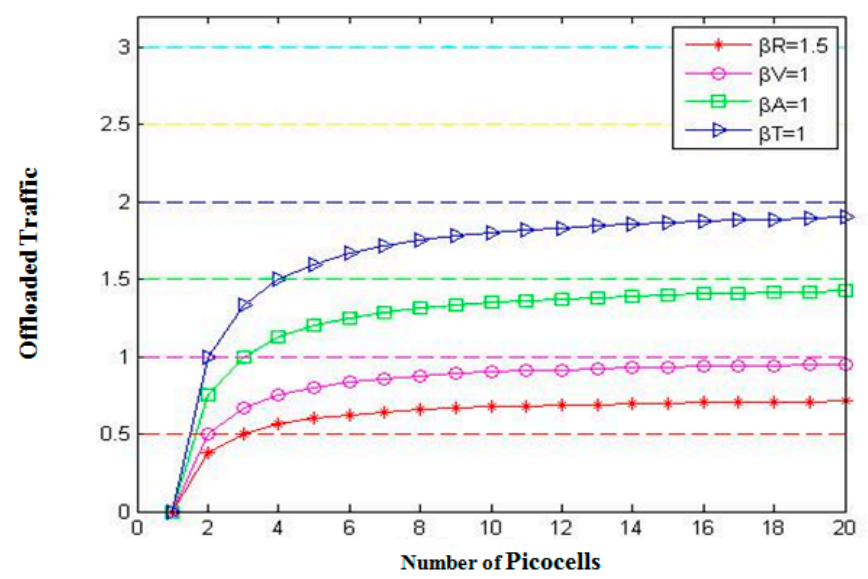

(a)

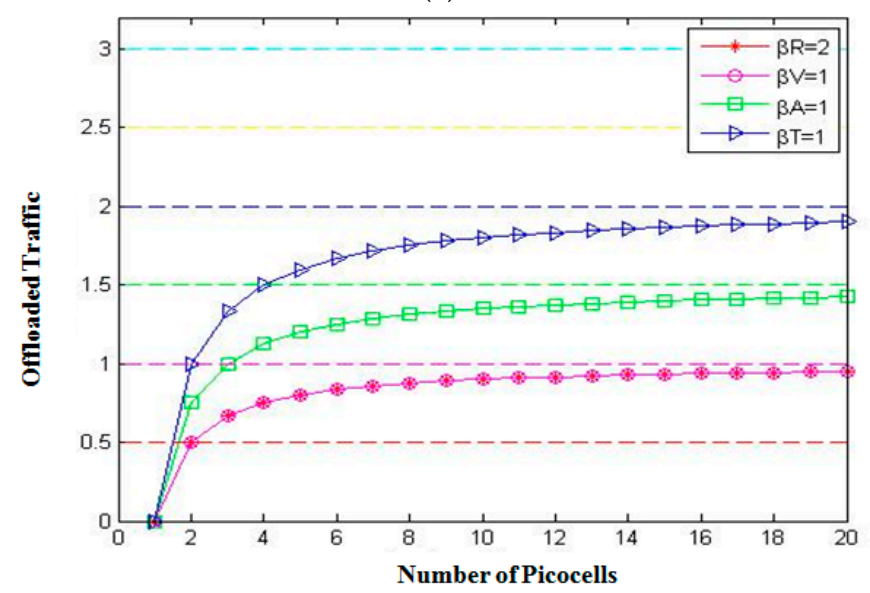

(b)

Figure 3. Offloaded traffic with (a) $\beta_{D}=4, \beta_{R}=1.5, \beta_{V}=1, \beta_{T}=1, \beta_{A}=1$ and (b) $\beta_{D}=5, \beta_{R}=2$, $\beta_{\mathrm{T}}=1, \beta_{\mathrm{A}}=1$, and $\beta_{\mathrm{V}}=1$. 
In Figure 4, the economic incentives were kept for all types of data equal to 1 except in (a) $\beta_{\mathrm{V}}=1.5$, and in (b) $\beta_{\mathrm{V}}=2$. It was observed that the video data got a higher rate of the download, and it matched with audio data at incentive $\beta_{\mathrm{A}}=1$ when we further increased the incentive for video data to 2 .

In Figure 5, the economic incentives were kept for all types of data equal to 1 except in (a) $\beta_{\mathrm{A}}=1.5$, and in (b) $\beta_{A}=2$. It was observed that the audio data got a higher rate of the download, and it even got higher than text data at incentive $\beta_{\mathrm{T}}=1$. When we further increased the incentive for audio data to 2 , i.e., $\beta_{\mathrm{A}}=2$, the download was further increased, and it matched with the highest download of text data, i.e., $\beta_{\mathrm{T}}=2$.

In Figure 6, the economic incentives are kept for all types of data equal to 1 except in (a) $\beta_{\mathrm{T}}=1.5$, and in (b) $\beta_{\mathrm{T}}=2$. It was observed that the video data got a higher rate of the download, and it matched with audio data at incentive $\beta_{\mathrm{A}}=1$. When incentives for text data were further increased to 2 , i.e., $\beta_{\mathrm{T}}=2$, the download was exponentially increased. In this case, the text data gave a greater response to the economic incentives as compared to other types of data.

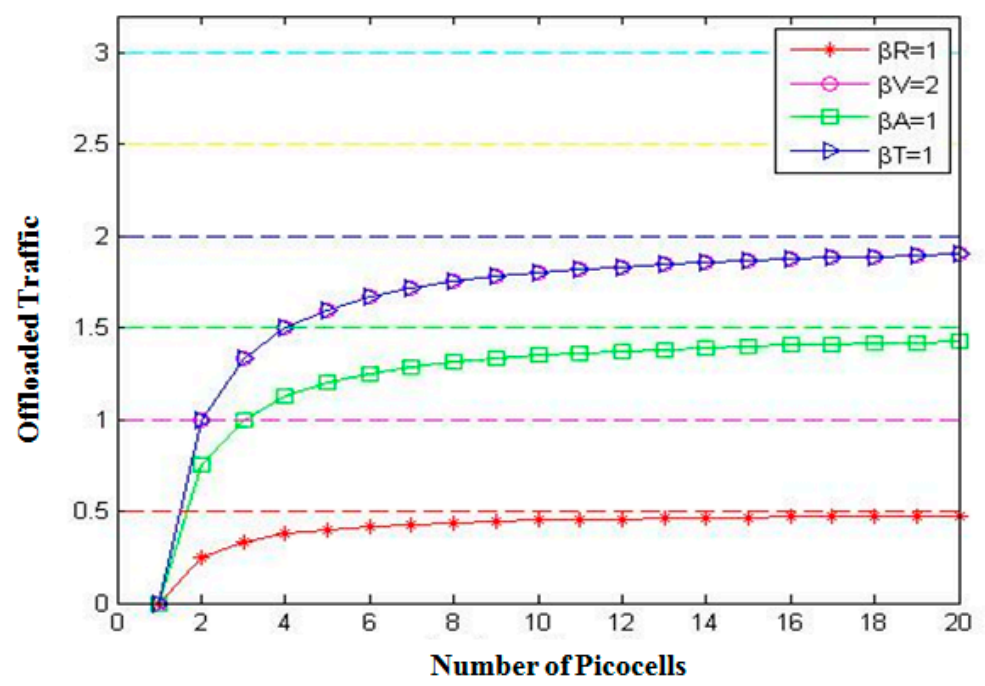

(a)

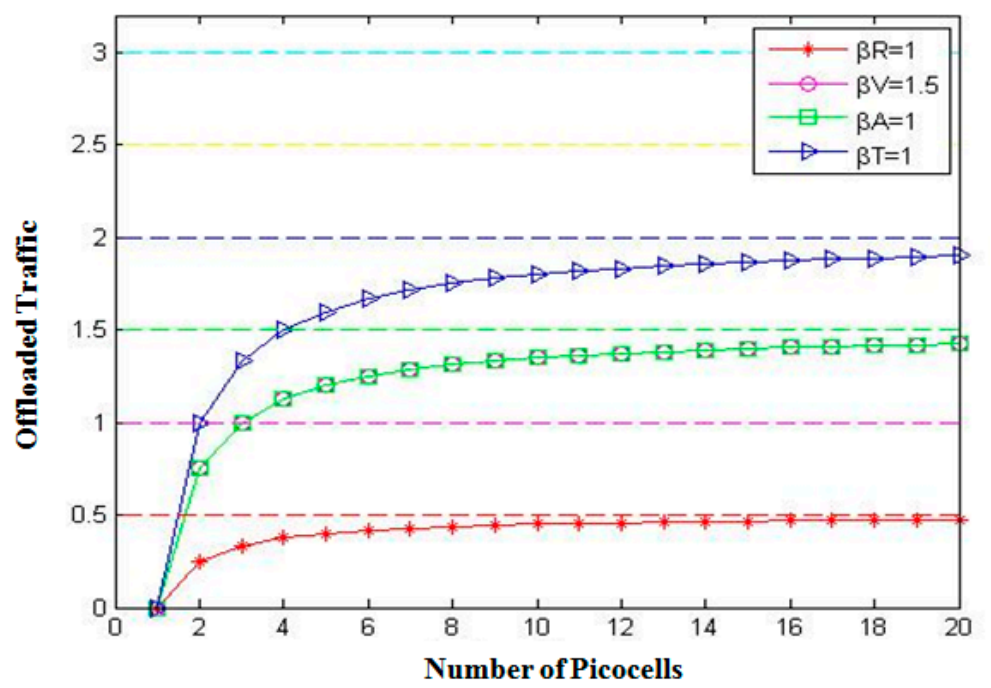

(b)

Figure 4. Offloaded traffic with (a) $\beta_{\mathrm{D}}=4.5, \beta_{\mathrm{V}}=1.5, \beta_{\mathrm{T}}=1, \beta_{\mathrm{A}}=1$ and $\beta_{\mathrm{R}}=1$, and $(\mathbf{b}) \beta_{\mathrm{D}}=5, \beta_{\mathrm{V}}=2$, $\beta_{\mathrm{T}}=1, \beta_{\mathrm{A}}=1$, and $\beta_{\mathrm{R}}=1$. 


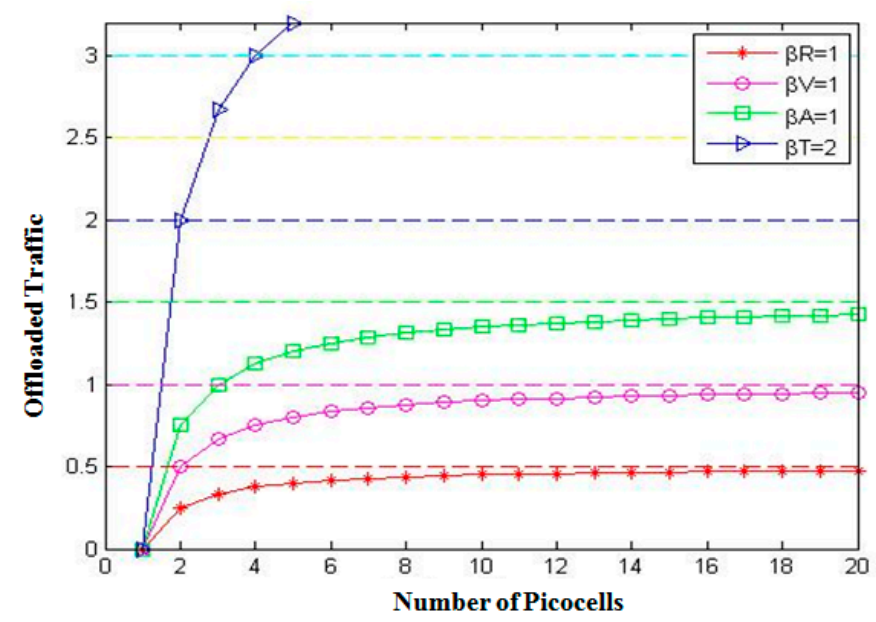

(a)

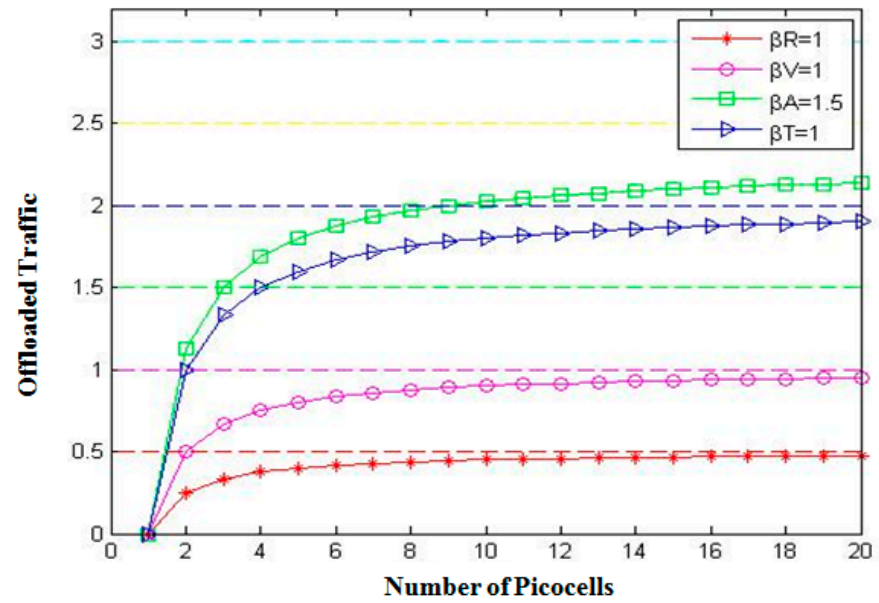

(b)

Figure 5. Offloaded traffic with (a) $\beta_{\mathrm{D}}=4.5, \beta_{\mathrm{V}}=1, \beta_{\mathrm{T}}=1, \beta_{\mathrm{A}}=1.5$ and $\beta_{\mathrm{R}}=1$, and $(\mathbf{b}) \beta_{\mathrm{D}}=5, \beta_{\mathrm{V}}=1$, $\beta_{\mathrm{T}}=1, \beta_{\mathrm{A}}=2$, and $\beta_{\mathrm{R}}=1$.

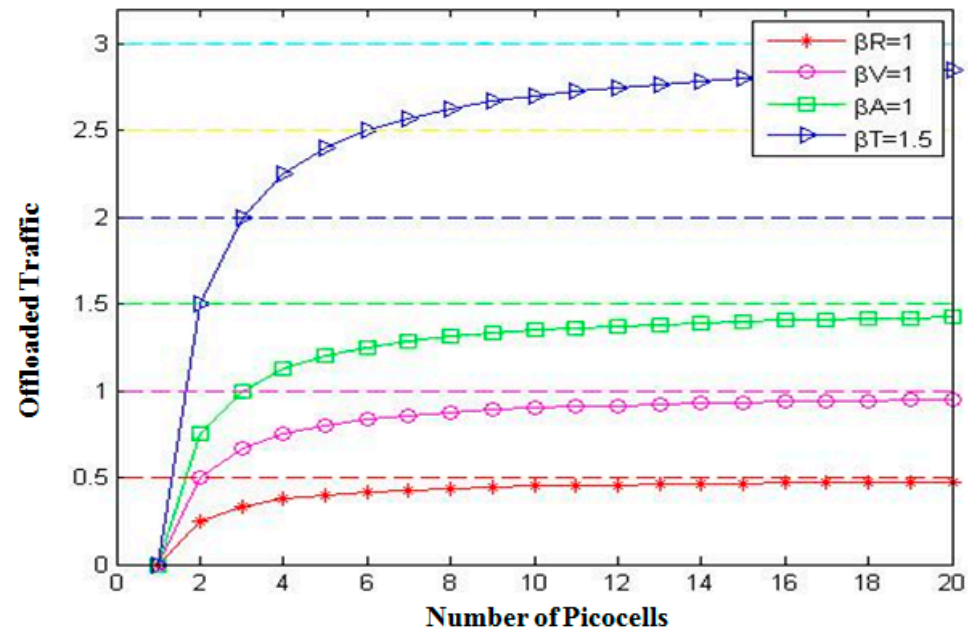

(a)

Figure 6. Cont. 


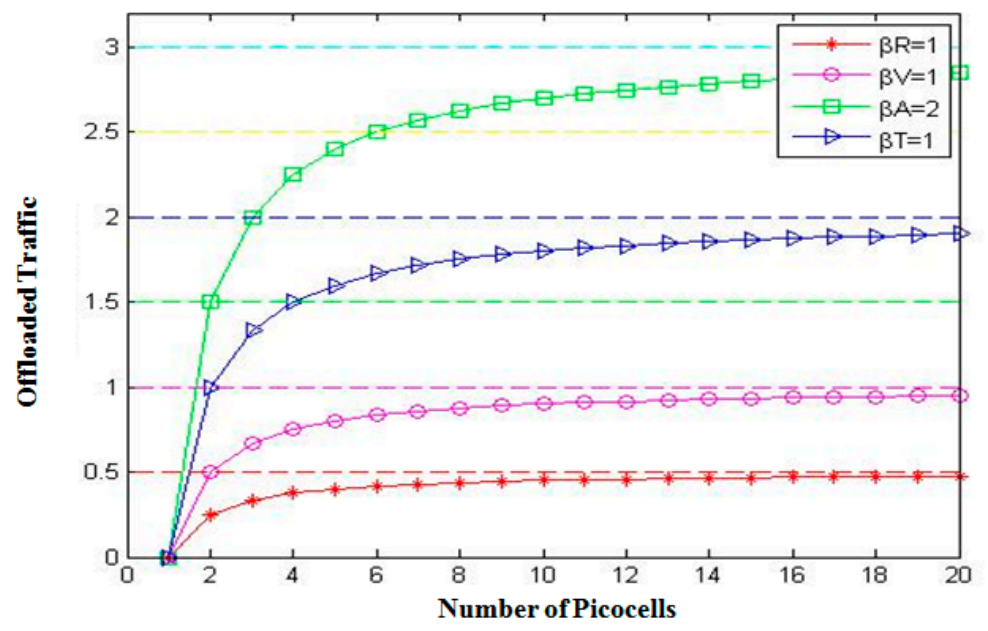

(b)

Figure 6. Offloaded traffic with (a) $\beta_{\mathrm{D}}=4.5, \beta_{\mathrm{V}}=1, \beta_{\mathrm{T}}=1, \beta_{\mathrm{A}}=1.5$ and $\beta_{\mathrm{R}}=1$, and $(\mathbf{b}) \beta_{\mathrm{D}}=5, \beta_{\mathrm{V}}=1$, $\beta_{\mathrm{T}}=1, \beta_{\mathrm{A}}=2$, and $\beta_{\mathrm{R}}=1$.

In the above examination, it was noticed that the rate of change of offloading of different data types based on the priority and the principle of natural understanding that which type was more convenient to allow directly at a particular stage and which was required to be offloaded.

The above experimental results show that the offloading effect of all types of the data depended on the various incentive rates.

\subsection{Effect of Changes in APs on Offloading}

In this section, the effect of a number of access points on the type of data downloads was investigated. In Figure 7, it is observed that the effect of varying the number of APs varied the download of different types of data. In Figure 8, the number APs were kept between 1 and 20 that were used to offload various data types to investigate the optimal number of $A P_{S}$ for offloading. To support an optimal number of $A P s$ of the network, we took the varying number of the APs in experiments and the significant increase in offloading various data types. This experiment found the optimal number of the $A P s$ in the networks for offloading.

When the number of $A P S$ was less than 5, significance increase found in the traffic and after greater than $5 A P s$ such as 6, 7 to 20 the effect gradually increased, but the sharp increase was noticed up to twenty picocells, while the change in case of accruing more $A P s$ from 5 to 20 , is comparatively minor.

The variation in offloading audio type data was observed much higher and ranges from about 1.4 to 2.9 for the given set of parameters. Audio type was the second priority data type in live to accommodate on the fastest primary channel. In the video traffic offloading of video data based on a predefined strategy for video offloading when live streaming, which required primary channel priority higher than audio or text because breakage in video streaming badly affected the audience interest; that was why we focused on such issues primarily to make a cost-effective solution. The textual data offloading varies highly, and the graph values range from 1.9 to 3.9 , which shows that textual data was less needy of fastest sources and even small breakages were un-noticeable or all pages loading at once was not necessary, therefore, secondary channels were more appropriate for text. 


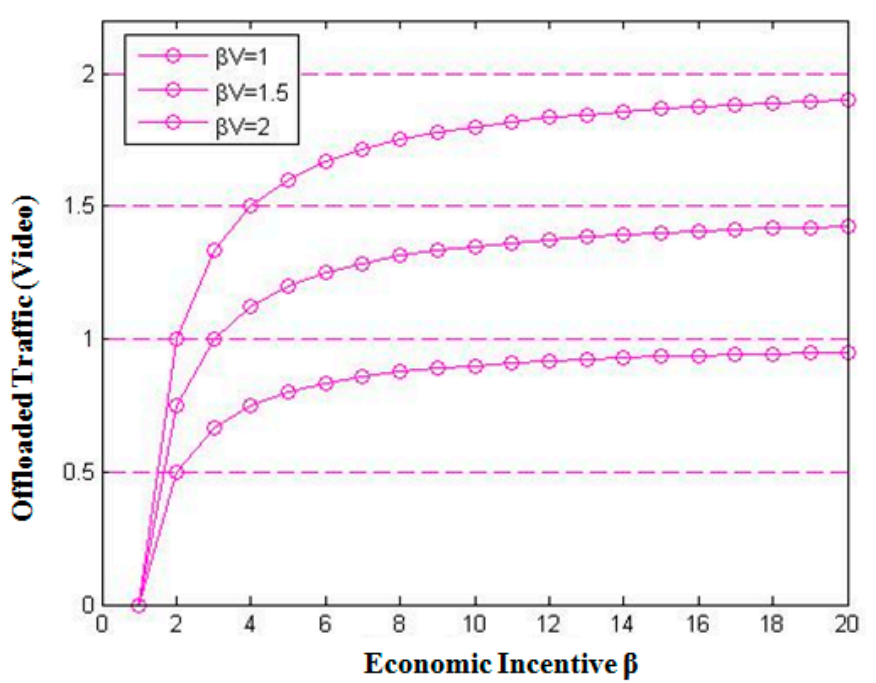

(a)

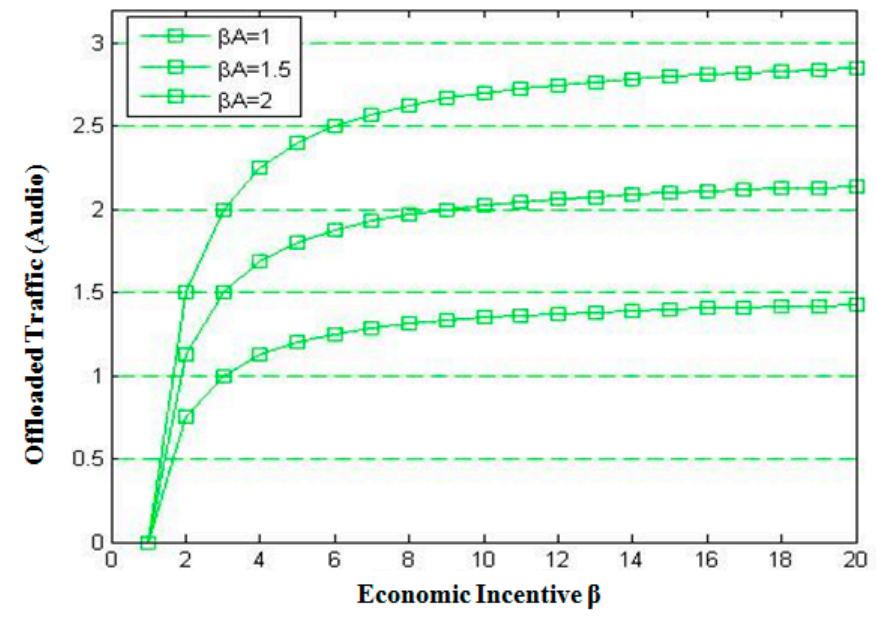

(b)

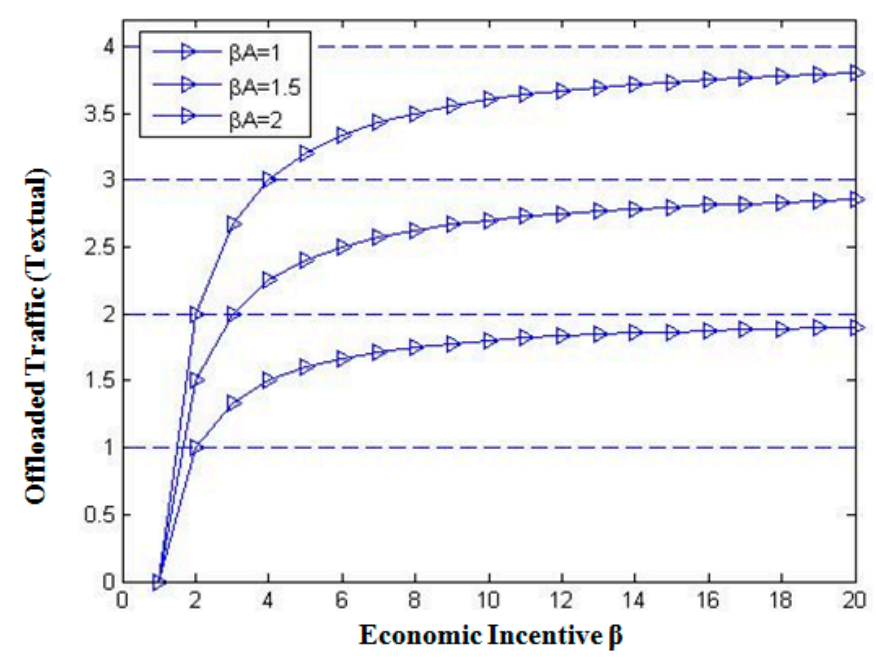

(c)

Figure 7. Effect of a number of multiple APs for offloading different types of data: (a) $\beta_{\mathrm{A}}$, (b) $\beta_{\mathrm{V}}$, and (c) $\beta_{\mathrm{T}}$. 


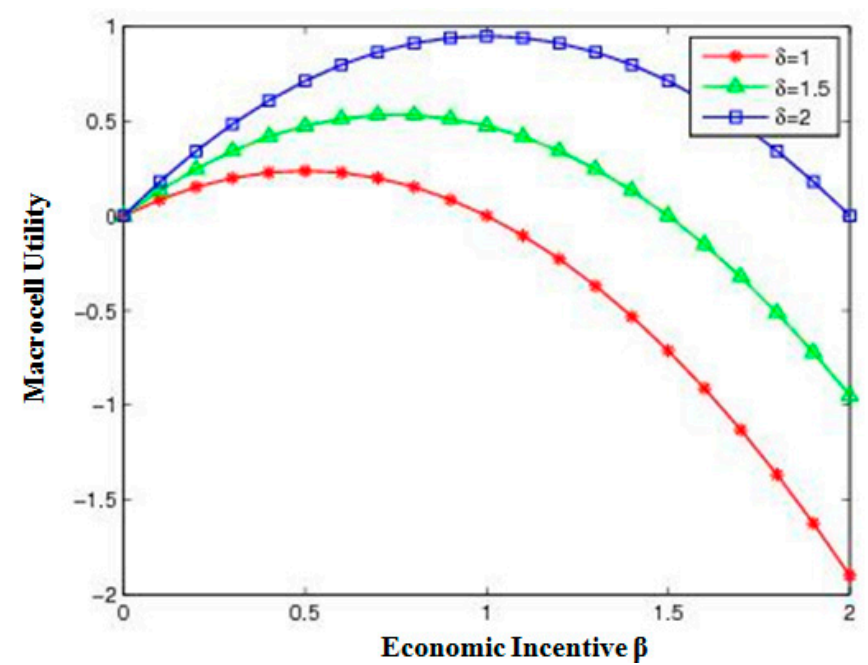

(a)

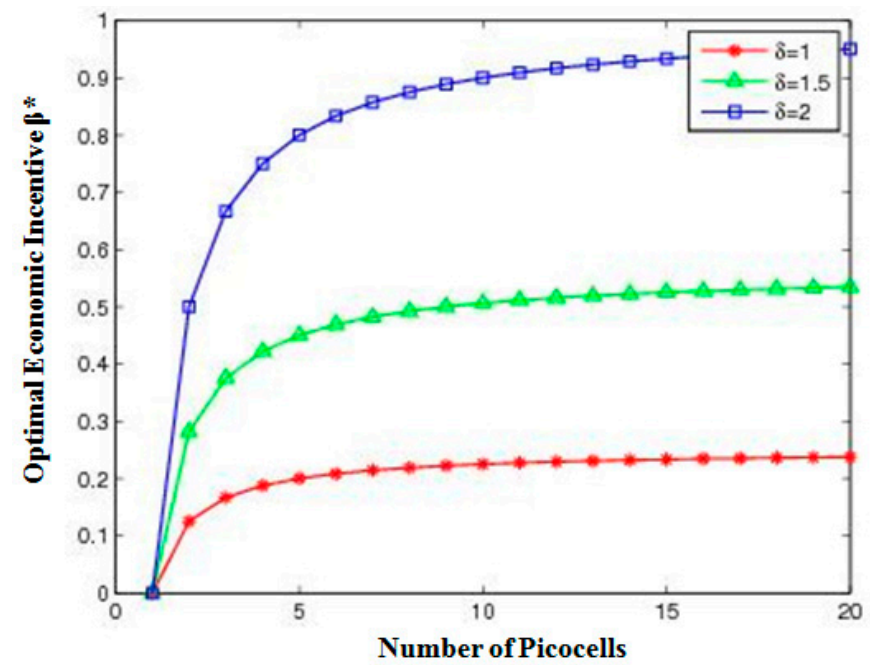

(b)

Figure 8. MBS's linear utility and linear cost: (a) Macrocell utility $\beta_{\mathrm{V}} \mathrm{s}$ economic incentive, and (b) optimal economic incentive $\beta_{\mathrm{V}}$ s number of $A P s$.

\section{2. $M B S^{\prime}$ Payoff}

This experiment showed that the MBS net profit was based on the economic incentive offered for various traffics in the folder of linear utility and linear cost, and three cases of unit spectrum saving. The graph showed that the lower value of $\delta$ give us a lesser value for $M B S$, and another graph showed the variation in economic incentive offered for various traffic types and varying the number of $A P s$. We found that optimal economic incentive increases significantly when the $A P S$ were less than 5 , and a regular increase from 6 to 20 APs.

\subsection{Comparative Analysis}

The proposed scheme, referred to as Game Theoretic Mobile Data Offloading (GMDO) in this section, has been compared with Economic Incentive for Data Offloading (EIDO) [30]. Data traffic for four major types of the applications, i.e., file backup, HTTP, video, and VoIP were considered in the experiments.

Figure 9 shows the performance of the twenty numbers of picocells and respective incentive offered, and our proposed model performed better as compared to the previous model, which is due to the high offloading ratio. 
Figure 10 shows the variation in the congestion level for the given line spectrum and a total of twenty picocells. At 5 picocells, the congestion control of EIDO dropped completely, while EIDO never drops to zero at 20 picocells.

Figure 11, we have tested our model for a given range of values. The results show better performance as compared to the existing model, EIDO. The proposed model proved to have better resource utilization. In other words, GMDO maintained a good level of QoS.

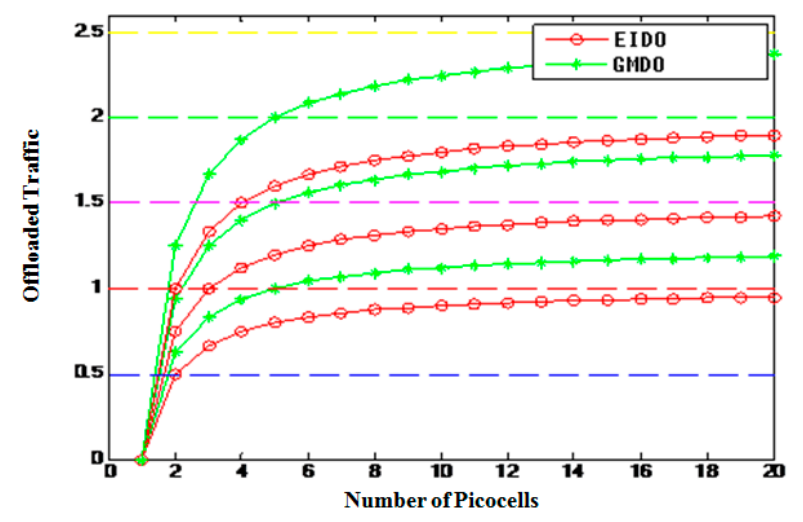

Figure 9. Offloading ratio evaluation.

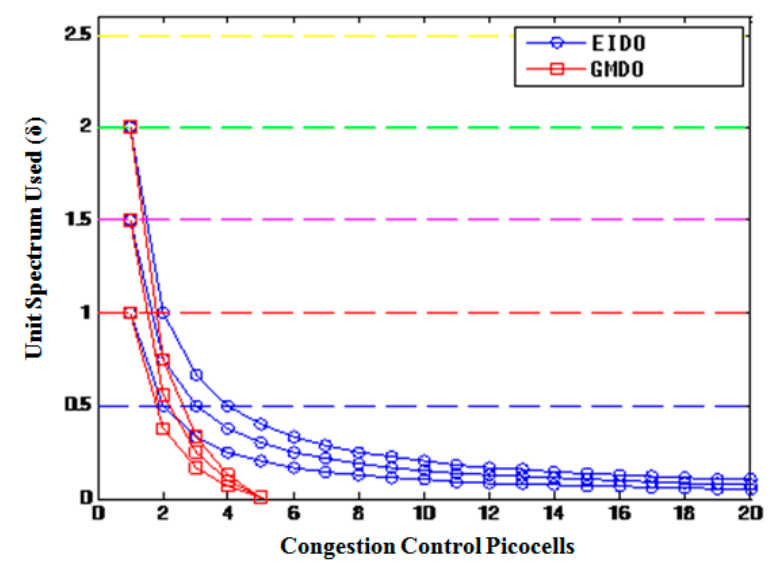

Figure 10. Congestion control evaluation.

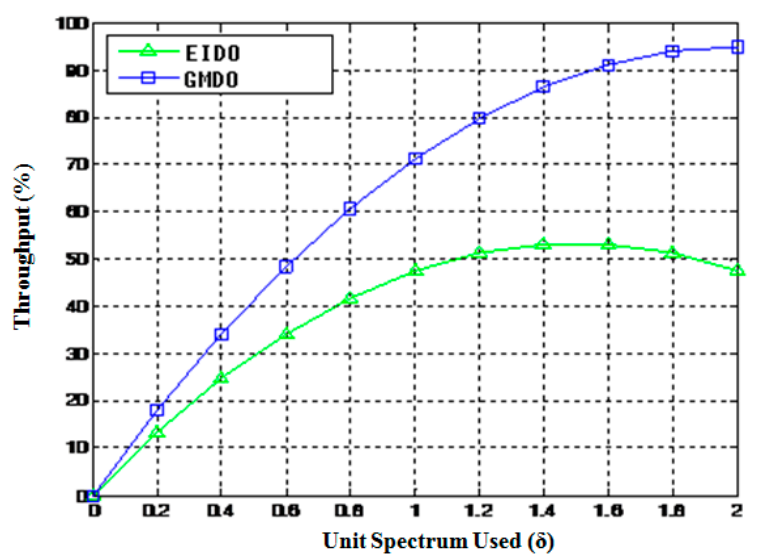

Figure 11. Throughput performance evaluation. 
Figure 12 shows that GMDO performed well in the reduction of network delay when compared with EIDO. This was due to the enhanced response to the end user, which is the primary goal of QoS.

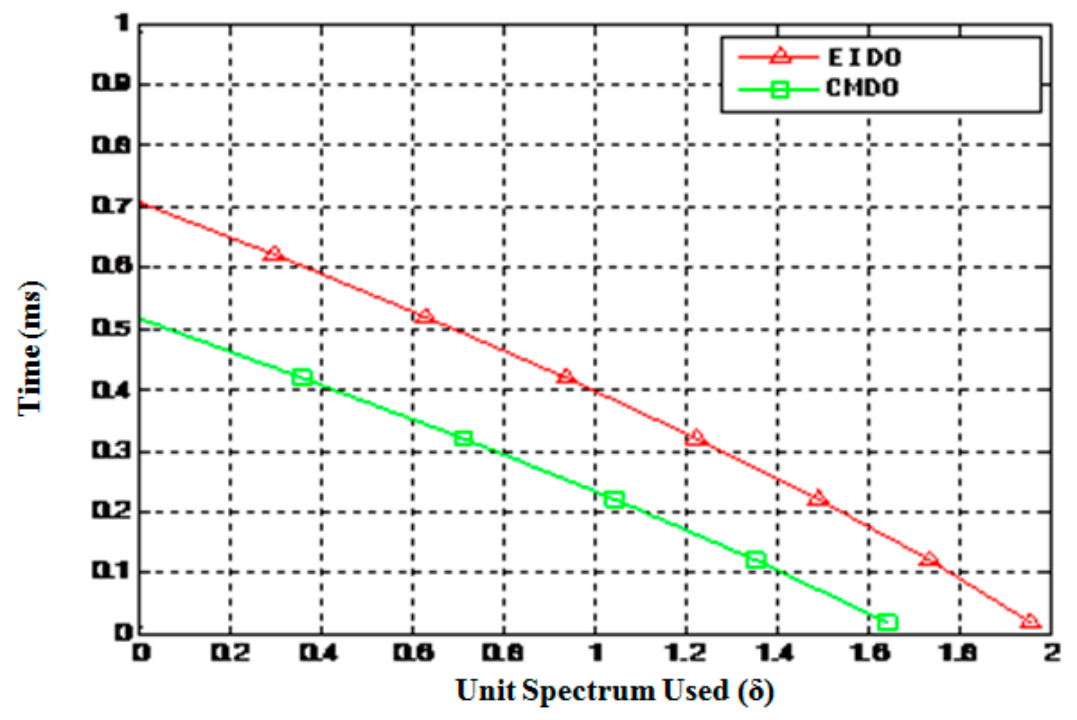

Figure 12. Delay performance evaluation.

\section{Conclusions}

In this work, we proposed an incentive framework based on the traffic types, such as real-time traffic, video traffic, audio traffic, and text traffic. The work kept the QoS requirements of each traffic based on game theory using a two-stage Stackelberg approach for solving the congestion problem in existing wireless cellular networks. We studied the economic dealings between the MBS and APS in mobile data offloading based on the traffic types. Our game model was based on the Stackelberg approach and our model worked in two phases. In the first phase, the MBS offered the economic incentives based on traffic types to the $A P S$, and in the second phase, the APs use this incentive and offload traffics. We have proved the mathematical model and existence of unique Nash Equilibrium. Numerical outcomes characterized a direct process of the best possible offloading ratio, and they legalized the efficiency of the proposed game-based offloading algorithm using a Stackelberg approach. We found that the proposed traffic-types-based game achieved a unique Stackelberg equilibrium. The simulation results showed the optimal incentive and the optimal number of APs for offloading in peak time, and showed that the proposed model significantly improved the MNO profit and APs offloading ratio for all types of traffic. The proposed schema achieved the supreme performance. This is the first time that an economic incentive scheme has been developed based on traffics priority in an overload problem. The proposed work has been compared with another work, EIDO. The comparative results show that the proposed work is much better in terms of throughput ( $50 \%$ higher), delay (overall $0.2 \mathrm{~ms}$ lower), congestion control ( $\delta=0.2$ higher), and offloading ratio ( $50 \%$ higher).

This work can be further expanded by the consideration of multi-base stations and multiple APs. In such scenarios, the mechanisms must also address various additional parameters, such as handoff and move speed, etc. Moreover, the handling of rational users having an adaptive interaction with the APs could also be integrated into such systems. An intelligent model will be needed to design the users' behavior and preferences. Additionally, an intelligent security mechanism in APS and $M B S$ communication can be incorporated into the systems by adopting a Stackelberg approach. The development of a fully-integrated network architecture for offloading that merges the various offloading proposals and management of unlicensed band for traffic offloading with mobility parameter are also open issues for future research. 
Author Contributions: M.A. and S.U.K. built the game based model for mobile data offloading. R.A. analyzes the game based model. M.A. designed the simulation and performed the experiments; S.U.K. analyzed the results; D.S. analyzed the data. M.A. wrote the paper; D.S. revised the paper. S.K. and R.A. supervise and mentored the work and reviewed the whole work and provided suggestions for the enrichment of the paper.

Funding: The research is funded by the Hankuk University of Foreign Studies research fund 2018. The Article Processing Charges (APC) would be funded by Research Fund \& Publication Committee (RFPC), Hankuk University of Foreign Studies research fund 2018.

Acknowledgments: This research work is supported by the Hankuk University of Foreign Studies research fund 2018.

Conflicts of Interest: The authors declare no conflict of interest.

\section{References}

1. Balen, J.; Zagar, D.; Viho, C.; Martinovic, G. Quality of Service in Wireless Sensor Networks: A Survey and Related Patents. Recent Pat. Comput. Sci. 2011, 4, 188-202.

2. Muñoz, L.R.; Mora, D.; Barriga, R. Teachers Cisco certification and their impact in Cisco Networking Academy program. Revista Científica de la UCSA 2017, 4, 50-56. [CrossRef]

3. Hossain, E.; Le, L.B.; Niyato, D. Wiley series on adaptive and cognitive dynamic systems. In Radio Resource Management in Multi-Tier Cellular Wireless Networks; John Wiley \& Sons Ltd: Hoboken, NJ, USA, 2013; pp. 326-327.

4. Cevik, T.; Gunagwera, A. Coverage and Energy Efficiency Optimization for Randomly Deployed Multi-Tier Wireless Multimedia Sensor Networks. Int. J. Commun. Netw. Inf. Secur. 2018, 10, 28-36.

5. Han, B.; Hui, P.; Kumar, V.S.A.; Marathe, M.V.; Shao, J.; Srinivasan, A. Mobile Data Offloading through Opportunistic Communications and Social Participation. IEEE Trans. Mob. Comput. 2012, 11, 821-834. [CrossRef]

6. Aijaz, A.; Aghvami, H.; Amani, M. A survey on mobile data offloading: Technical and business perspectives. IEEE Wirel. Commun. 2013, 20, 104-112. [CrossRef]

7. Rebecchi, F.; de Amorim, M.D.; Conan, V.; Passarella, A.; Bruno, R.; Conti, M. Data Offloading Techniques in Cellular Networks: A Survey. IEEE Commun. Surv. Tutor. 2015, 17, 580-603. [CrossRef]

8. Lee, K.; Lee, J.; Yi, Y.; Rhee, I.; Chong, S. Mobile Data Offloading: How Much Can WiFi Deliver? IEEE/ACM Trans. Netw. 2013, 21, 536-550. [CrossRef]

9. Ajinegoro, R.M.N.; Perdana, D.; Negara, M.R. Performance Analysis of Mobility Impact on IEEE 802.11ah Standard with Traffic Pattern Scheme. Int. J. Commun. Netw. Inf. Secur. 2018, 10, 139-147.

10. Dimatteo, S.; Hui, P.; Han, B.; Li, V.O.K. Cellular Traffic Offloading through WiFi Networks. In Proceedings of the 2011 IEEE Eighth International Conference on Mobile Ad-Hoc and Sensor Systems, Valencia, Spain, 17-22 October 2011.

11. Kang, X.; Chia, Y.K.; Sun, S.; Chong, H.F. Mobile data off-loading through a third-party WiFi access point: An operator's perspective. IEEE Trans. Wirel. Commun. 2014, 13, 5340-5351. [CrossRef]

12. Chen, Q.; Yu, G.; Maaref, A.; Li, G.; Huang, A. Rethinking Mobile Data Offloading for LTE in Unlicensed Spectrum. IEEE Trans. Wirel. Commun. 2016, 15, 4987-5000. [CrossRef]

13. Lee, J.; Yi, Y.; Chong, S.; Jin, Y. Economics of WiFi Offloading: Trading Delay for Cellular Capacity. IEEE Trans. Wirel. Commun. 2014, 13, 1540-1554. [CrossRef]

14. Zhang, H.; Bennis, M.; DaSilva, L.A.; Han, Z. Multi-leader multi-follower Stackelberg game between Wi-Fi, small cell, and macrocell networks. In Proceedings of the 2014 IEEE Global Communications Conference, Austin, TX, USA, 8-12 December 2014.

15. Wang, K.; Lau, F.C.M.; Chen, L.; Schober, R. Pricing Mobile Data Offloading: A Distributed Market Framework. IEEE Trans. Wirel. Commun. 2016, 15, 913-927. [CrossRef]

16. Wang, K.; Lau, F.C.M.; Chen, L.; Schober, R. An incentive framework for cellular traffic offloading. IEEE Trans. Mob. Comput. 2014, 13, 541-555.

17. Shah-Mansouri, H.; Wong, V.W.S.; Huang, J. An Incentive Framework for Mobile Data Offloading Market under Price Competition. IEEE Trans. Mob. Comput. 2017, 16, 2983-2999. [CrossRef]

18. Zhang, H.; Jiang, C.; Beaulieu, N.C.; Chu, X.; Wang, X.; Quek, T.Q.S. Resource Allocation for Cognitive Small Cell Networks: A Cooperative Bargaining Game Theoretic Approach. IEEE Trans. Wirel. Commun. 2015, 14, 3481-3493. [CrossRef] 
19. Zhang, H.; Dong, Y.; Cheng, J.; Hossain, M.J.; Leung, V.C.M. Fronthauling for 5G LTE-U Ultra Dense Cloud Small Cell Networks. IEEE Wirel. Commun. 2016, 23, 48-53. [CrossRef]

20. Xu, X.; Yuan, C.; Li, J.; Zhang, H.; Tao, X. Reverse Auction Based Green Offloading Scheme for Small Cell Heterogeneous Networks. Mob. Inf. Syst. 2016, 2016, 1-10. [CrossRef]

21. Park, Y.; Kim, S. Game-based data offloading scheme for IoT system traffic congestion problems. EURASIP J. Wirel. Commun. Netw. 2015, 2015. [CrossRef]

22. Aijaz, A.; Uddin, N.; Holland, O.; Aghvami, A.H. On Practical Aspects of Mobile Data Offloading to Wi-Fi Networks. arXiv, 2015.

23. Iosifidis, G.; Gao, L.; Huang, J.; Tassiulas, L. A Double-Auction Mechanism for Mobile Data-Offloading Markets. IEEE/ACM Trans. Netw. 2015, 23, 1634-1647. [CrossRef]

24. Gao, L.; Iosifidis, G.; Huang, J.; Tassiulas, L.; Li, D. Bargaining-Based Mobile Data Offloading. IEEE J. Sel. Areas Commun. 2014, 32, 1114-1125. [CrossRef]

25. Oliveira, R.P.; Góis, L.A.; Foronda, A. Enhanced PF Scheduling Algorithm for LTE Networks. Int. J. Commun. Netw. Inf. Secur. 2018, 10, 49-55.

26. Isnawati, A.F.; Hidayat, R.; Sulistyo, S.; Mustika, I.W. A Novel Utility Function of Power Control Game in Multi-Channel Cognitive Femtocell Network. Int. J. Commun. Netw. Inf. Secur. 2017, 9, 405-411.

27. Wang, C.; Liang, C.; Yu, F.R.; Chen, Q.; Tang, L. Computation Offloading and Resource Allocation in Wireless Cellular Networks with Mobile Edge Computing. IEEE Trans. Wirel. Commun. 2017, 16, 4924-4938. [CrossRef]

28. Wang, C.; Liang, C.; Yu, F.R.; Chen, Q.; Tang, L. Joint computation offloading, resource allocation and content caching in cellular networks with mobile edge computing. In Proceedings of the 2017 IEEE International Conference on Communications (ICC), Paris, France, 21-25 May 2017.

29. Tsiropoulou, E.E.; Kapoukakis, A.; Papavassiliou, S. Uplink resource allocation in SC-FDMA wireless networks: A survey and taxonomy. Comput. Netw. 2016, 96, 1-28. [CrossRef]

30. Ho, T.M.; Tran, N.H.; Do, C.T.; Kazmi, S.M.A.; LeAnh, T.; Hong, C.S. Data offloading in heterogeneous cellular networks: Stackelberg game-based approach. In Proceedings of the 2015 17th Asia-Pacific Network Operations and Management Symposium (APNOMS), Busan, Korea, 19-21 August 2015.

(C) 2018 by the authors. Licensee MDPI, Basel, Switzerland. This article is an open access article distributed under the terms and conditions of the Creative Commons Attribution (CC BY) license (http:/ / creativecommons.org/licenses/by/4.0/). 